\title{
Numerical Experiments with a Symmetric High-Resolution Shock-Capturing Scheme
}

\section{H.C. Yee}

(NASA-TH-88325) NOMERTCAL EXPERIHENTS YITH A SYMAETRIC HIGH-RESOLUTION SHOCK-CAPTOBING SCHEHE (AASA) $10 \mathrm{p}$ AVail: NTIS HC A02/HF A01 CSCL $12 \mathrm{~A}$ 


\section{ERRATA}

NASA Technical Memorandum 88325, June, 1986

Numerical Experiments with a Symmetric

High-Resolution Shock-Capturing Scheme

H.C. Yee

Pages 5: Figure 4, "Mach contours" should read "Pressure contours". 


\section{Numerical Experiments with a Symmetric High-Resolution Shock-Capturing Scheme}

H. C. Yee, Ames Research Center, Moffett Field, California

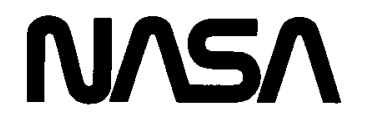

National Aeronautics and

Space Administration

Ames Research Center

Moffett Field, California 94035 


\title{
NUMERICAL EXPERIMENTS WITH A SYMMETRIC HIGH-RESOLUTION SHOCK-CAPTURING SCHEME ${ }^{\dagger}$
}

\author{
H.C. $\mathrm{YEE} \ddagger$ \\ MS 202A-1, NASA Ames Research Center \\ Moffett Field, CA. 94035 USA
}

\section{Introduction}

Characteristic-based explicit and implicit total variation diminishing (TVD) schemes for the twodimensional compressible Euler equations have recently been developed $[1,2]$. This is a generalization of recent work of Roe and Davis $[3,4]$ to a wider class of symmetric (non-upwind) TVD schemes other than Lax-Wendroff. Roe and Davis's schemes can be viewed as a subset of the class of explicit methods. The main properties of the present class of schemes are that they can be implicit, and, when steady-state calculations are sought, the numerical solution is independent of the time step. In reference [2], a comparison of a linearized form of the present implicit symmetric TVD scheme with an implicit upwind TVD scheme originally developed by Harten $[5,6]$ and modified by Yee [7] was given. The results favored the symmetric method. It was found that the symmetric method is just as accurate as the upwind method while requiring less computational effort. It is emphasized that the generic use of the notion upwind and symmetric TVD schemes here pertains to the schemes without the limiter present. With a limiter present, an upwind TVD scheme no longer has its traditional upwinding meaning. The same situation also applies to symmetric TVD schemes. Another way of distinguishing an upwind from a symmetric TVD scheme is that the numerical dissipation term corresponding to an upwind TVD scheme is upwind weighted [5-10], as opposed to the numerical dissipation term corresponding to a symmetric TVD scheme which is centered [1-4].

Currently, more numerical experiments are being conducted on time-accurate calculations and on the effect of grid topology, numerical boundary condition procedures, and different flow conditions on the behavior of the method for steady-state applications. The purpose of this paper is to report our experiences with this type of scheme and give some basic guidelines for using the scheme. A description of upwind and symmetric TVD schemes, including formulation and extension to the two-dimensional Euler equations of gas dynamics in curvilinear coordinates, can be found in references $[2,7,11]$. This paper contains a brief description of the TVD algorithm and a summary of the numerical computations.

\section{Description of Algorithm in One Dimension}

Consider a one-dimensional system of hyperbolic conservation laws

$$
\frac{\partial U}{\partial t}+\frac{\partial F(U)}{\partial x}=0
$$

Here $U$ and $F(U)$ are column vectors of $m$ components and $A=\partial F / \partial U$. Let the eigenvalues of $A$ be $\left(a^{1}, a^{2}, \ldots, a^{m}\right)$. Denote $R$ as the matrix whose columns are eigenvectors of $A$, and $R^{-1}$ as the inverse of $R$. Let the grid spacing be denoted by $\Delta x$ such that $x=j \Delta x$, and let $U_{j+\frac{1}{2}}$ denotes some symmetric average of $U_{j}$ and $U_{j+1}$ (for example, $U_{j+\frac{1}{2}}=\frac{1}{2}\left(U_{j+1}+U_{j}\right)$ ). Let $a_{j+\frac{1}{2}}^{l}, R_{j+\frac{1}{2}}, R_{j+\frac{1}{2}}^{-1}$ denote the quantities $a^{l}, R$, $R^{-1}$ related to $A$ evaluated at $U_{j+\frac{1}{2}}$. Define

$$
\alpha_{j+\frac{1}{2}}=R_{j+\frac{1}{2}}^{-1}\left(U_{j+1}-U_{j}\right)
$$

as the difference of the local characteristic variables.

A one-parameter family of conservative explicit and implicit second-order TVD schemes can be written as

$$
U_{j}^{n+1}+\lambda \theta\left[\tilde{H}_{j+\frac{1}{2}}^{n+1}-\tilde{H}_{j-\frac{1}{2}}^{n+1}\right]=U_{j}^{n}-\lambda(1-\theta)\left[\tilde{H}_{j+\frac{1}{2}}^{n}-\tilde{H}_{j-\frac{1}{2}}^{n}\right]
$$

tPreprint for proceedings of the Tenth International Conference on Numerical Methods in Fluid Dynamics, June 23-27, 1986, Beijing, China.

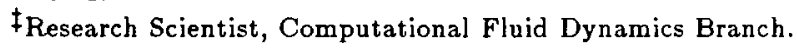


where $\lambda=\frac{\Delta t}{\Delta x}, \Delta t$ is the time step and $\theta$ is a parameter. When $\theta \neq 0$, the scheme is implicit. The numerical flux function $\tilde{H}_{j+\frac{1}{2}}$ can be expressed as

$$
\tilde{H}_{j+\frac{1}{2}}=\frac{1}{2}\left[F_{j}+F_{j+1}+R_{j+\frac{1}{2}} \Phi_{j+\frac{1}{2}}\right] .
$$

The elements of the $\Phi_{j+\frac{1}{2}}$ denoted by $\left(\phi_{j+\frac{1}{2}}^{l}\right)^{S}$ for a general second-order symmetric TVD scheme [1] are

$$
\left(\phi_{j+\frac{1}{2}}^{l}\right)^{S}=-\lambda \beta\left(a_{j+\frac{1}{2}}^{l}\right)^{2} \hat{Q}_{j+\frac{1}{2}}^{l}-\psi\left(a_{j+\frac{1}{2}}^{l}\right)\left[\alpha_{j+\frac{1}{2}}^{l}-\hat{Q}_{j+\frac{1}{2}}^{l}\right],
$$

where $\alpha_{j+\frac{1}{2}}^{l}$ are elements of (2). When $\beta=1$, the method is best suited for time-accurate calculations, and when $\beta=0$, it is mainly for steady-state applications. The function $\psi$ is

$$
\psi(z)= \begin{cases}|z| & |z| \geq \epsilon \\ \left(z^{2}+\epsilon^{2}\right) / 2 \epsilon & |z|<\epsilon\end{cases}
$$

Here $\psi(z)$ in (4b) is an entropy correction to $|z|$ where $\epsilon$ is a small positive parameter (see reference [12] for a formula for $\epsilon$ ). Examples of the 'limiter' function $\hat{Q}_{j+\frac{1}{2}}^{l}$ can be expressed as

$$
\begin{aligned}
& \hat{Q}_{j+\frac{1}{2}}^{l}=\operatorname{minmod}\left(\alpha_{j-\frac{1}{2}}^{l}, \alpha_{j+\frac{1}{2}}^{l}\right)+\operatorname{minmod}\left(\alpha_{j+\frac{1}{2}}^{l}, \alpha_{j+\frac{3}{2}}^{l}\right)-\alpha_{j+\frac{1}{2}}^{l} \\
& \hat{Q}_{j+\frac{1}{2}}^{l}=\operatorname{minmod}\left(\alpha_{j-\frac{1}{2}}^{l}, \alpha_{j+\frac{1}{2}}^{l}, \alpha_{j+\frac{3}{2}}^{l}\right) \\
& \hat{Q}_{j+\frac{1}{2}}^{l}=\operatorname{minmod}\left[2 \alpha_{j-\frac{1}{2}}^{l}, 2 \alpha_{j+\frac{1}{2}}^{l}, 2 \alpha_{j+\frac{3}{2}}^{l}, \frac{1}{2}\left(\alpha_{j-\frac{1}{2}}^{l}+\alpha_{j+\frac{3}{2}}^{l}\right)\right] .
\end{aligned}
$$

The minmod function of a list of arguments is equal to the smallest number in absolute value if the list of arguments is of the same sign, or is equal to zero if any arguments are of opposite sign.

The elements of the $\Phi_{j+\frac{1}{2}}$ denoted by $\left(\phi_{j+\frac{1}{2}}^{l}\right)^{U}$ for a second-order upwind TVD scheme, originally developed by Harten, and later modified and generalized by the author $[1,13]$, are

$$
\left(\phi_{j+\frac{1}{2}}^{l}\right)^{U}=\sigma\left(a_{j+\frac{1}{2}}^{l}\right)\left(g_{j+1}^{l}+g_{j}^{l}\right)-\psi\left(a_{j+\frac{1}{2}}^{l}+\gamma_{j+\frac{1}{2}}^{l}\right) \alpha_{j+\frac{1}{2}}^{l} .
$$

The function $\sigma(z)=\frac{1}{2} \psi(z)+\left(\theta-\frac{1}{2}\right) \lambda \beta z^{2}$ and

$$
\gamma_{j+\frac{1}{2}}^{l}=\sigma\left(a_{j+\frac{1}{2}}^{l}\right)\left\{\begin{array}{ll}
\left(g_{j+1}^{l}-g_{j}^{l}\right) / \alpha_{j+\frac{1}{2}}^{l} & \alpha_{j+\frac{1}{2}}^{l} \neq 0 \\
0 & \alpha_{j+\frac{1}{2}}^{l}=0
\end{array} .\right.
$$

Examples of the 'limiter' function $g_{j}^{l}$ can be expressed as

$$
\begin{aligned}
& g_{j}^{l}=\operatorname{minmod}\left(\alpha_{j-\frac{1}{2}}^{l}, \alpha_{j+\frac{1}{2}}^{l}\right) \\
& g_{j}^{l}=\left(\alpha_{j+\frac{1}{2}}^{l} \alpha_{j-\frac{1}{2}}^{l}+\left|\alpha_{j+\frac{1}{2}}^{l} \alpha_{j-\frac{1}{2}}^{l}\right|\right) /\left(\alpha_{j+\frac{1}{2}}^{l}+\alpha_{j-\frac{1}{2}}^{l}\right) \\
& g_{j}^{l}=S \cdot \max \left\{0, \min \left(2\left|\alpha_{j+\frac{1}{2}}^{l}\right|, S \cdot \alpha_{j-\frac{1}{2}}^{l}\right), \min \left(\left|\alpha_{j+\frac{1}{2}}^{l}\right|, 2 S \cdot \alpha_{j-\frac{1}{2}}^{l}\right)\right\} ; S=\operatorname{sgn}\left(\alpha_{j+\frac{1}{2}}^{l}\right) .
\end{aligned}
$$

The method of extending scalar TVD schemes to nonlinear systems of hyperbolic conservation laws (1) in equation (3) is sometimes referred as the local characteristic approach and is a variant of Roe's linear Riemann solver [14]. The advantages of this approach as opposed to Davis's simplified approach [4] to systems are that: (a) this approach in effect uses scalar schemes on each characteristic field; (b) the limiter used need not be the same for each field; e.g., one can use a more compressive limiter for the linear fields and use a less compressive limiter for the nonlinear fields; (c) one can even use different schemes for different fields; (d) it is more efficient than the exact or approximate Riemann solvers; (e) it provides a natural way to linearize the implicit TVD schemes. For the one-dimensional Euler equation of gas dynamics, the characteristic fields consist of two nonlinear fields $u \pm c$ and a linear field $u$. 
For two-dimensional time-accurate calculations, the explicit scheme $\beta=1, \theta=0$ is implemented by the Strang type of time splitting method [15]. For steady-state numerical study, the implicit scheme $\beta=0, \theta=1$ considered here is implemented in a conservative noniterative ADI form [2]. The numerical solution is independent of the time step. The implicit operator has a regular block tridiagonal structure and the resulting block tridiagonal matrix is diagonally dominant. One can modify a classical ADI central difference code by simply changing the linear numerical dissipation term into the one designed for the TVD scheme; i.e., the third term of equation (3b). See references $[7,11]$ for more details.

Let the grid indexing at the left boundary in the $x$-direction be $j=0$. For the explicit operator, this is a five-point scheme in each coordinate direction and one needs values of $g_{0}^{l}$ as well as $U_{0}$. For simplicity and illustration purposes, the values of $U_{0}$ are assumed to be updated by the procedure described in reference [11]. The following three ways of obtaining $g_{j}^{l}$ at the boundaries are discussed.

$$
g_{0}^{l}=0 ; \quad g_{0}^{l}=g_{1}^{l} ; \quad g_{0}^{l}=\alpha_{\frac{3}{2}}^{l} .
$$

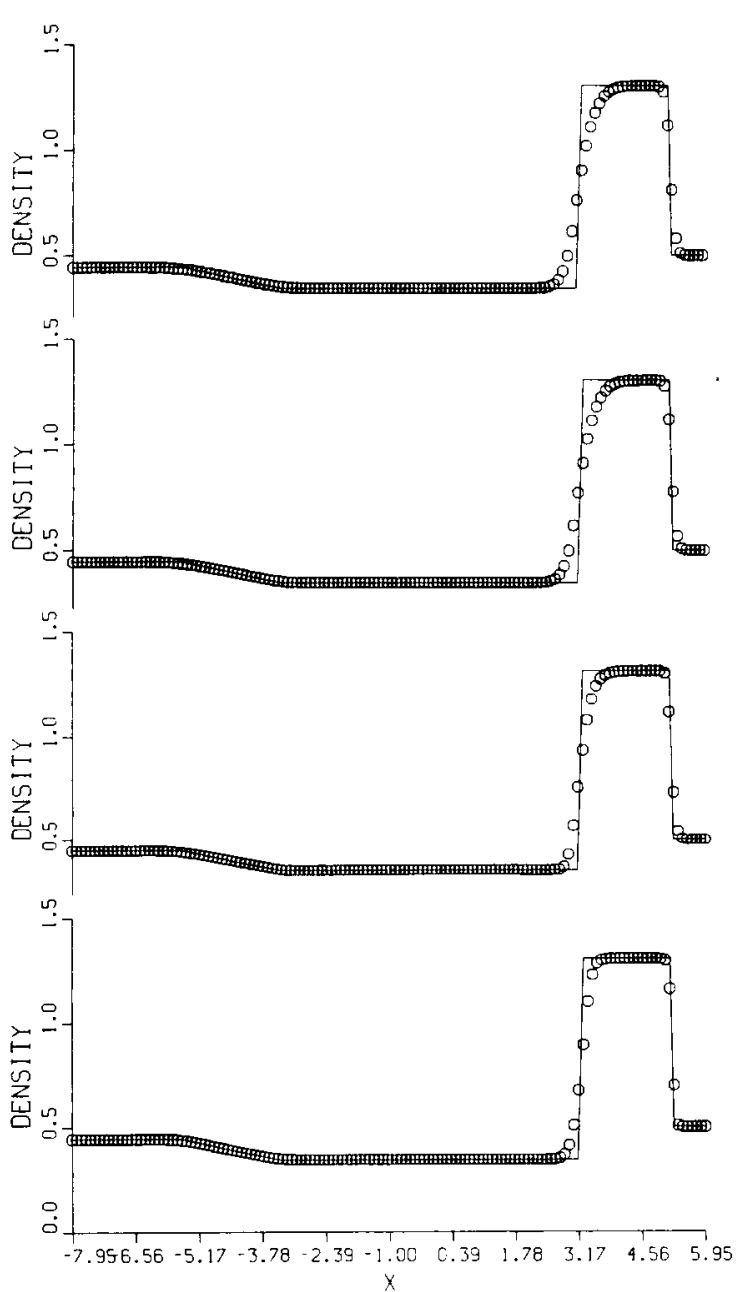

Fig. 1 The Density Distribution (- exact, o computed) of the shock tube problem using limiter $(4 \mathrm{c}-4 \mathrm{e})$ for the symmetric scheme and using (5d) for the upwind scheme (top to bottom)
One can interpret (6a) as a first-order TVD scheme at the boundary, whereas $(6 \mathrm{~b})$ is obtained by zeroth-order extrapolation. Boundary scheme (6c) is the least dissipative among the three. It is obtained by simply removing the logic in the limiter and using the next availiable $\alpha^{l}$.

\section{Numerical Results}

3.1 Time Accurate Calculations: The numerical experiments were mainly performed on the one-dimensional shock tube problem and the NACA0012 and NACA0018 airfoils. Limiter (4e) produces slightly sharper shocks and contact discontinuities than (4c) or (4d).

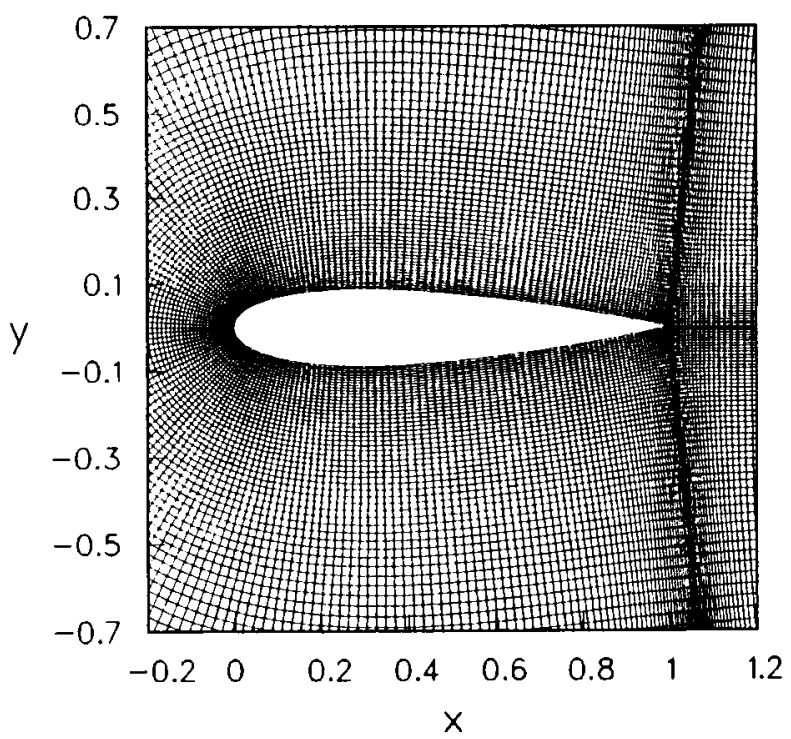

Fig. 2 The $299 \times 79 \mathrm{C}$ grid for the NACA0018 airfoil. 
One Dimension: Figure 1 shows the density distribution for the same shock tube problem as used in reference [5]. The solid lines are the exact solutions and the circles are the results computed by using limiters (4c-4e) for the symmetric TVD scheme and (5d) for the upwind scheme respectively (from top to bottom). With the use of the same limiter, the study shows that in general the symmetric scheme is slightly more diffusive than the upwind scheme. However, the resolution (not shown) of the symmetric scheme with limiter (4e) is better than the upwind scheme with limiter $(5 c)$. Note that not all of the limiters that are designed for upwind schemes are applicable for symmetric schemes.

Two Dimensions: In the experiment, a time dependent curved shock wave collides with the NACA0018 airfoil at an angle of attack $\alpha=30^{\circ}$. The Mach number $M_{S}$ is approximately 1.5 at the moment of collision. The experiment was performed by Mandella and Bershader [16] of Stanford University. In the numerical simulations, a constant planar incident shock of $M_{S}=1.5$ and $\alpha=30^{\circ}$ is used as an initial condition just before the leading edge of the airfoil. This work was done jointly with Young Moon of U.C. Berkeley. Figure 2 shows the $299 \times 79 \mathrm{C}$ grid for the NACA0018 airfoil. Figure 3 shows the numerical results in the form of density contour plots using (4e) with the symmetric scheme, and (5d) for the nonlinear fields and (5e) for the linear fields with the upwind scheme at (approximately) the four sequential instances of the interferograms. The incident and reflected shocks, Mach stems, and slip surface on the lower surface are captured within 3 grid points. Also the vortices at the upper nose and the trailing edge of the airfoil are well captured in the simulation. On the upper surface the experimental results show a weak slip surface which it is not captured in the simulation. By increasing the grid resolution around that region, this slip surface is also expected to be captured. Even though a planar shock was used to model the curved shock, the shape and location of the discontinuities compare favorably with the experiments. The shock resolution of the symmetric TVD scheme is similar to the upwind scheme except that it is slightly more diffusive. This leads us to believe that even closer agreement between the numerical simulation and the experiments is possible if a time dependent curved shock is used. A detail description of the physical problem and a more closely simulated result with the exact experiment can be found in a paper by Moon and Yee [17]. Aside from the difference in CPU time (at least $35-40 \%$ less), one advantage of symmetric TVD schemes over upwind schemes is that the symmetric TVD schemes are less sensitive to numerical boundary condition treatments for higher Mach number flows. Figure 4 shows the computations of the symmetric TVD scheme (4e) at a CFL number of 0.98 for a planar incident shock of $M_{S}=20$ and $\alpha=30^{\circ}$. With the same numerical boundary conditions, the upwind scheme diverges; i.e., a characteristic type of boundary condition is needed for the upwind scheme.

3.2 Steady-State Calculations: Some steady-state computations have been carried out to illustrate the sensitivity of the implicit symmetric (and upwind) scheme on the various numerical boundary condition procedures, grid topologies and flow conditions. A typical solution computed by the symmetric scheme using (4e) is shown in figure 5. Figure 6 shows the $163 \times 49 \mathrm{C}$ grid with the outer boundary 24 chord lengths away from the body. Some of the numerical study using (4c) and (4d) with $\beta=0$ and $\theta=1$ using the same grid is summarized below.

Accuracy: To demonstrate how numerical boundary conditions affect the accuracy of the scheme, the computations of the NACA0012 airfoil at a freestream Mach number $M_{\infty}=0.8$ and $\alpha=1.25^{\circ}$ with (4c) are shown in figure 7. This figure compares the Mach contours of boundary schemes (6a) with (6b). One can see a drastic difference in resolution near the solid body. Away from the body, the resolution is indistinguishable. A comparison was also made between boundary schemes (6b) and (6c). No visible difference in accuracy was found.

Stability: Computation of the NACA0012 airfoil with freestream Mach numbers ranging from 0.8 to 1.8 with and without lift show that boundary schemes (6a) and (6b) have a similar stability and convergence rate. However boundary scheme (6c) has a lower stability bound than (6a) and (6b). This is due to the fact that boundary scheme $(6 \mathrm{c})$ is the least dissipative among the three procedures.

Grid Topology: The grid shown in figure 1 was generated by a hyperbolic grid method. This grid is fairly regular and for the most part is nearly orthogonal. The present method appears to have no difficulty in obtaining a fairly accurate solution. If one were to use a highly irregular and nonorthogonal grid, the present scheme would require a long time to reach steady state or, in an extreme case, fail to converge.

Convergence Rate: A numerical study was also conducted on the effect of a freestream Mach number on the convergence rate of the scheme. The following is based on $0.7 \leq M_{\infty} \leq 1.8,0^{\circ} \leq \alpha \leq 7^{\circ}$, and grid sizes ranging from $163 \times 33$ to $249 \times 49$ with either a $\mathrm{C}$ or $\mathrm{O}$ grid. For $0.7 \leq M_{\infty} \leq 0.8$, a $L_{2}$-norm residual of 

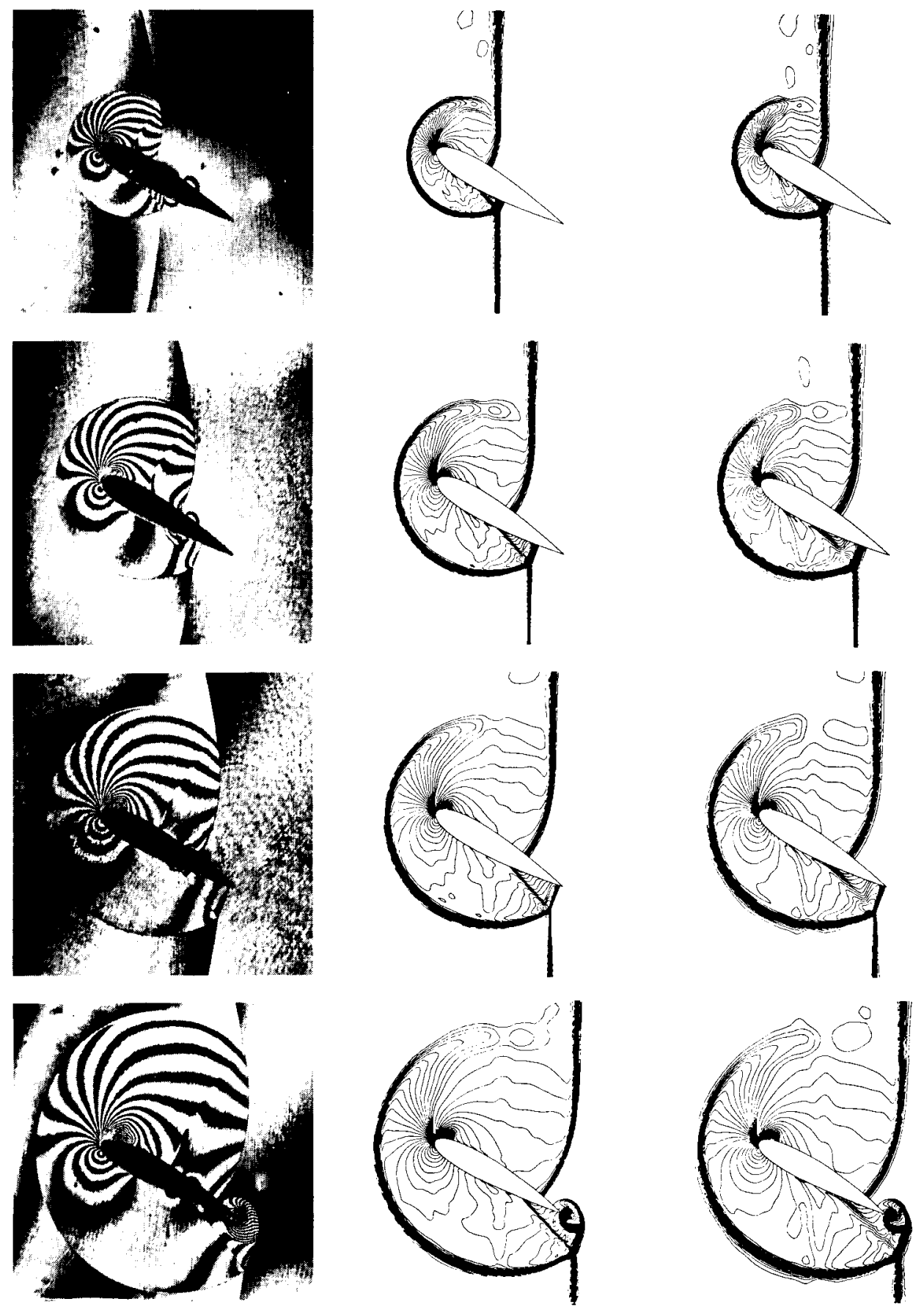

Fig. 3 The Density contours by the upwind scheme (5a,d,e) (middle) and by the symmetric scheme $(4 \mathrm{a}, \mathrm{e})$ (right) for the NACA0018 airfoil with $M_{S}=1.5$, $\alpha=30^{\circ}$ compared with the interferograms at approximately the same sequential instances.
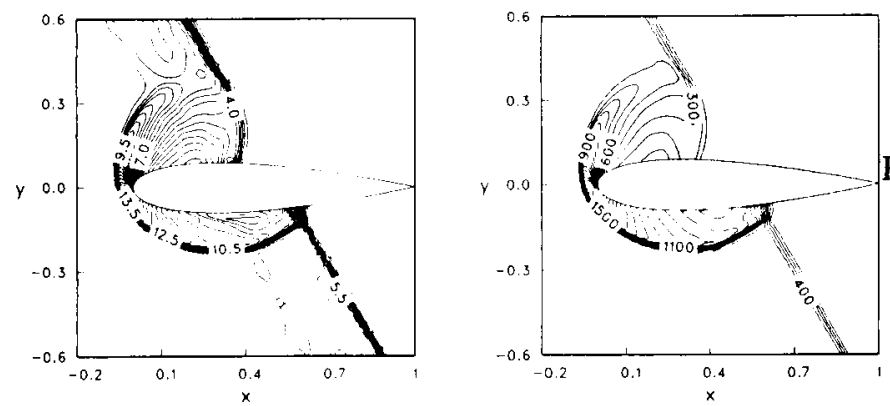

Fig. 4 The density contours (left) and Mach contours (right) by the symmetric scheme $(4 \mathrm{a}, \mathrm{e})$ for the NACA0018 airfoil with $M_{S}=$ $20, \alpha=30^{\circ}$. 

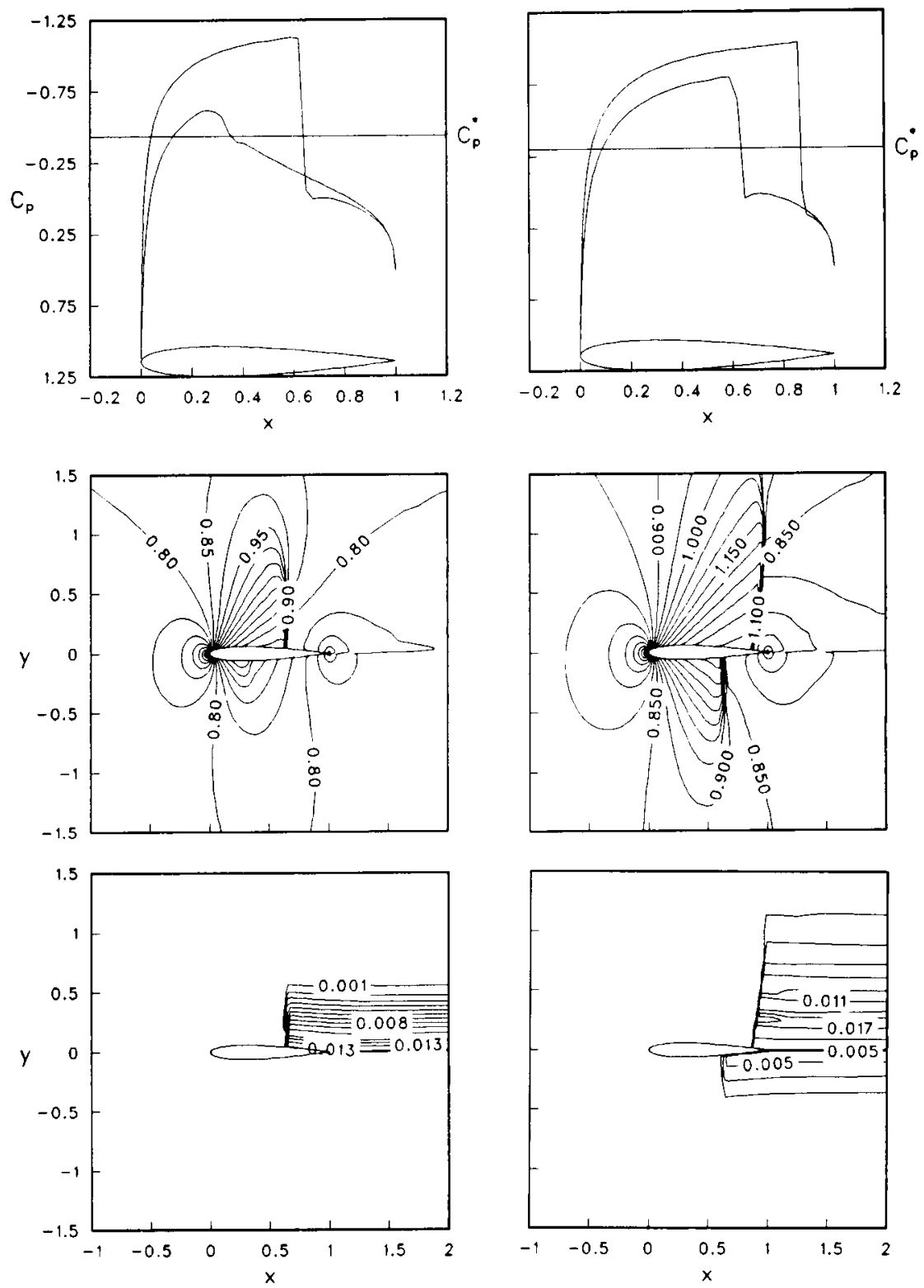

Fig. 5 The pressure coefficients, Mach contours and entropy contours (top to bottom) computed by the symmetric scheme $(4 \mathrm{a}, \mathrm{e})$ for the NACA0012 airfoil with $M_{\infty}=0.8, \alpha=1.25^{\circ}$ (left) and $M_{\infty}=0.85, \alpha=1^{\circ}$ (right).

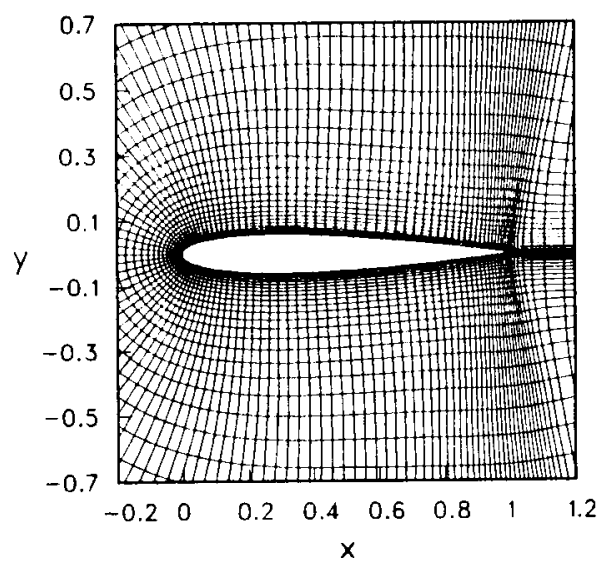

Fig. 6 The $163 \times 49 \mathrm{C}$ grid for the NACA0012 airfoil. 

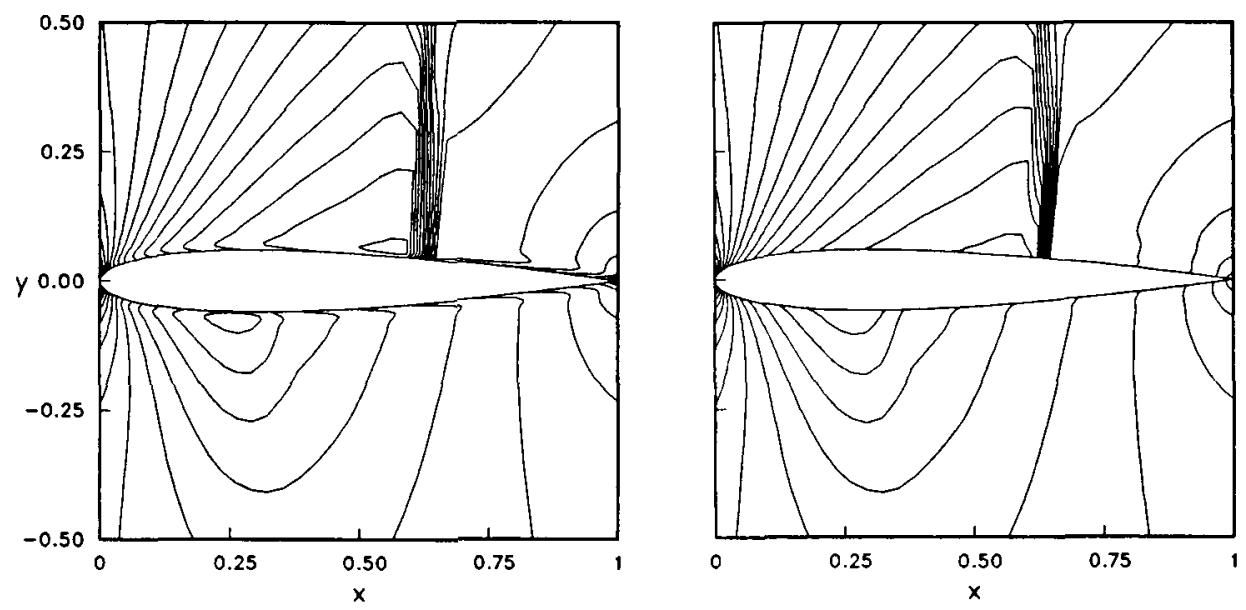

Fig. 7 The Mach contours computed by boundary schemes (6a) (left) and (6b) (right) for the NACA0012 airfoil with $M_{\infty}=0.8, \alpha=1.25^{\circ}$.

$10^{-7}$ can be reached in 600-1200 steps depending on the grid size, time step and $\alpha$. For $0.85 \leq M_{\infty} \leq 0.98$, a residual of $10^{-7}$ can be reached in 1500-3500 steps. For $1.2 \leq M_{\infty} \leq 1.8$, a residual of $10^{-7}$ can be reached in 200-500 steps.

\section{Concluding Remarks}

For problems containing shocks only, numerical experiments for steady-state calculations show that the implicit symmetric TVD schemes, while requiring less computational effort, are just as accurate as the implicit upwind TVD scheme originally developed by Harten [5] and modified by Yee [7]. However, for problems containing both shocks and contact discontinuities, numerical experiments show that the explicit symmetric TVD schemes are slightly more diffusive than the explicit upwind TVD scherne especially at the contact surfaces. The current study on the various steady-state airfoil calculations also indicates the degree of sensitivity of the method to grid topology, freestream Mach number, and angle of attack. Moreover, proper numerical boundary condition procedures are essential for numerical simulation of fluid flow. Improper treatment of boundary conditions can lead to instability and inaccuracy, even if one starts with a stable high-resolution scheme that catered to problems with shocks. Furthermore, for certain flow regimes, different linearization or relaxation procedures other than ADI should be investigated to improve the convergence rate of steady-state calculations.

\section{References}

1 H.C. Yee, NASA-TM 86775, July 1985, also to appear, J. Comput. Phys., 1986.

2 H.C. Yee, Proc. 6th GAMM Conf. Numer. Meth. in Fluid Mechanics, W. Germany, Sept. 1985.

3 P.L. Roe, ICASE Report No. 84-53, October 1984.

4 S.F. Davis, ICASE Report No. 84-20, June 1984.

5 A. Harten, NYU Report, Oct., 1982; SIAM J. Num. Anal, Vol. 21, 1984, pp. 1-23.

6 A. Harten, J. Comput. Phys., Vol. 49, 1983, pp 357-393.

7 H.C. Yee, Int. J. Comput. Math. Appl., in press.

8 P.L. Roe, AMS publications, Lectures in Appl. Math., Vol. 22, 1985.

9 P.K. Sweby, SIAM J. Num. Analy., Vol. 21, 1984.

10 S. Osher and S. Chakravarthy, SIAM J. Num. Analy., Vol. 21, 1984.

11 H.C. Yee and A. Harten, AIAA Paper No. 85-1513, July 1985, to appear AIAA J.

12 A. Harten and J.M. Hyman, J. Comp. Phys., Vol. 50, 1983.

13 H.C. Yee, "A comparative Study of Flux Limiters for Two-Dimensional Time-Accurate Calculations," in preparation.

14 P.L. Roe, J. Comp. Phys. Vol. 43, 1981.

15 H.C. Yee and P. Kutler, NASA TM-85845, August, 1983.

16 M. Mandella and D. Bershader, "Generation and Aerodynamic Interaction of Compressible Vortices," in preparation.

17 Y. J. Moon and H.C. Yee, "Numerical Simulation by TVD Schemes of Complex Shock Reflections from Airfoils at High Angle of Attack," in preparation. 


\begin{tabular}{|c|c|}
\hline $\begin{array}{l}\text { 1. Report No. } \\
\text { NASA TM-88325 }\end{array}$ & 3. Recipient's Catalog No. \\
\hline \multirow{2}{*}{$\begin{array}{l}\text { 4. Title and Subute } \\
\text { NUMERICAL EXPERIMENTS WITH A SYMMETRIC HIGH- } \\
\text { RESOLUTION SHOCK-CAPTURING SCHEME }\end{array}$} & $\begin{array}{l}\text { 5. Report Date } \\
\text { June } 1986\end{array}$ \\
\hline & 6. Performing Organization Code \\
\hline $\begin{array}{l}\text { 7. Author(s) } \\
\text { H. C. Yee }\end{array}$ & $\begin{array}{l}\text { 8. Performing Organization Report No. } \\
86316\end{array}$ \\
\hline \multirow{2}{*}{$\begin{array}{l}\text { 9. Pertorming Organization Name and Address } \\
\text { Ames Research Center } \\
\text { Moffett Field, CA } 94035\end{array}$} & 10. Work Unit No. \\
\hline & 11. Contract or Grant No. \\
\hline \multirow{2}{*}{$\begin{array}{l}\text { 12. Sponsoring Agencr Name and Address } \\
\text { National Aeronautics and Space Administration } \\
\text { Washington, DC } 20546\end{array}$} & $\begin{array}{l}\text { 13. Type of Report and Period Covered } \\
\text { Technical Memorandum }\end{array}$ \\
\hline & $\begin{array}{l}\text { 14. Sponsaring Agency Code } \\
505-31-01-01-00-21\end{array}$ \\
\hline \multicolumn{2}{|c|}{$\begin{array}{ll}\text { 15. Supplementary Notes } & \\
\text { Point of Contact: } & \text { H. C. Yee, Ames Research Center, M/S 202A-1 } \\
& \text { Moffett Field, CA } 94035 \text { (415) } 694-5548 \text { or FTS } 464-5548\end{array}$} \\
\hline \multicolumn{2}{|l|}{ 16. Abstract } \\
\hline \multicolumn{2}{|c|}{$\begin{array}{l}\text { Characteristic-based explicit and implicit total variation diminishing } \\
\text { (TVD) schemes for the two-dimensional compressible Euler equations have } \\
\text { recently been developed. This is a generalization of recent work of Roe } \\
\text { and Davis to a wider class of symmetric (non-upwind) TVD schemes other than } \\
\text { Lax-Wendroff. Roe and Davis's schemes can be viewed as a subset of the } \\
\text { class of explicit methods. The main properties of the present class of } \\
\text { schemes are that they can be implicit, and, when steady-state calculations } \\
\text { are sought, the numerical solution is independent of the time step. In a } \\
\text { recent paper, a comparison of a linearized form of the present implicit } \\
\text { symmetric TVD scheme with an implicit upwind TVD scheme originally } \\
\text { developed by Harten and modified by Yee was given. The results favored the } \\
\text { symmetric method. It was found that the symmetric method is just as accu- } \\
\text { rate as the upwind method while requiring less computational effort. Cur- } \\
\text { rently, more numerical experiments are being conducted on time-accurate } \\
\text { calculations and on the effect of grid topology, numerical boundary condi- } \\
\text { tion procedures, and different flow conditions on the behavior of the } \\
\text { method for steady-state applications. The purpose of this paper is to } \\
\text { report our experiences with this type of scheme and give some basic guide- } \\
\text { lines for using the scheme. }\end{array}$} \\
\hline \multicolumn{2}{|l|}{$\begin{array}{l}\text { 11. Key word (Suggested by Author(s) Numerical method, } \\
\text { Finite difference method, Computational } \\
\text { fluid dynamics, System of hyperbolic } \\
\text { conservation laws, Weak solutions, } \\
\text { Shock capturing, Conservative differ- } \\
\text { encing, TVD schemes, Implicit methods }\end{array}$} \\
\hline \begin{tabular}{|l|l|} 
19. Security Clossit. lot this reportl & 20. Security Classit. lot this pogel \\
Unclassified & Unclassif ied
\end{tabular} & \begin{tabular}{|c|r|} 
21. No. of Prges & 22. Price \\
10 & $\mathrm{AO2}$
\end{tabular} \\
\hline
\end{tabular}

-For sale by the National Tochnical Information Service, Springfield, Virginis 22161 